American Journal of Applied Sciences 2 (11): 1529-1532, 2005

ISSN 1546-9239

(c) 2005 Science Publications

\title{
Low Cost Long Distance Detector for Explosives and Chemical Analysis by IEC Application
}

\author{
${ }^{1}$ George H. Miley, ${ }^{2}$ Heinrich Hora, ${ }^{3}$ Frederick Osman, ${ }^{1}$ Yang Yang \\ ${ }^{1}$ Linchun Wu, ${ }^{1}$ Hiromo Momota and ${ }^{4}$ Xing Zhong Li \\ ${ }^{1}$ Department of Nuclear, Plasma and Radiological Engineering, University of Illinois, Urbana, IL. USA \\ ${ }^{2}$ Department of Theoretical Physics, University of New South Wales, Sydney 2052, Australia \\ ${ }^{3}$ School of Quantitative Methods and Mathematical Science, University of Western Sydney \\ Penrith-South 1797, Australia \\ ${ }^{4}$ Department of Modern Physics, Tsinghua University, Beijing, China
}

\begin{abstract}
A radiation source for detecting specific chemicals at several meter distances even behind walls, car doors or other barriers is the application of Million electron Volts (MeV) neutrons from nuclear fusion reactions at such low intensities to avoid any danger for human bodies. The chemical analysis consists in the neutron activation of nuclei emitting then gamma radiation of lines very specific for the excited nuclei. The neutron generation by the Inertial Electrostatic Confinement (IEC) had been developed to a level where very low cost neutron generators in mass production may be developed with a power supply from a normal AC plug-in or a battery. For specific chemicals e.g. the ratio of nitrogen against other elements used in all explosives, the selection of few specific gamma lines for the detectors may be of sufficiently low cost in the case of mass production.
\end{abstract}

Key words: Neutron activation analysis, nuclear fusion, gamma line spectroscopy, chemical analysis, detection of explosives

\section{INTRODUCTION}

It is a long desire how to detect explosives in suicide belts of passengers or the larger amount of explosives in cars passing street corners by using a low cost mass-produced detection system. The chemical neutrons activation analysis is a well-known method used in advanced laboratories where, however, expensive neutron sources and very complicate gamma spectrometers for quantitative measurements of all kinds of elements with their myriads of spectral lines are being used. For reducing the analysis to few elements only, e.g. the nitrogen against another to detect chemical explosives (or fertilizers) carried around or packed in motor cars at distances up to ten and more meters, a simplified version of the gamma line detectors has to be developed for low cost mass production and a very low cost MeV neutron source is needed.

Using the fusion reaction of heavy hydrogen (deuterium D) involves no safety problems and results in neutrons of $2.4 \mathrm{MeV}$ energy. For a longer distance detection of e.g. for checking large and compact containers, the $14 \mathrm{MeV}$ neutron from the reaction of $\mathrm{D}$ with the super-heavy hydrogen tritium (T) is interesting. A problem is then only that the detectors must not be destroyed avoiding that the small amount of tritium is released to the environment. The level of neutron production in continuous or pulsed operation of the neutron can be left at such low level that no danger for human bodies is implied. The level of these reactions is so low that - regrettably - any exothermic fusion energy production is excluded by numerous orders of magnitude.

For the fusion reactions at such a low intensity level, there exist several options as a linear pulsed gas discharge with up to 100.000 Volts, or the plasma focus. A further option is the inertial electrostatic confinement, IEC, about which the following achievements are summarized in the following with the prospects for a development of the neutron source for low cost mass production. An advantage of these developments is given from the possible application of the IEC for a very safe nuclear fission reactor ${ }^{[1]}$ where based on developments discussed by Dautray and Rubbia as competition by IEC $^{[2-4]}$, the present research reactor Garching II could have used the fast switching and unlimited operated IEC with the same neutron fluxes as the very slow switched and frequently regenerated $\mathrm{Cf} 251$ neutron source.

\section{THE IEC CONCEPT}

The Inertial Electrostatic Confinement (IEC) concept dates back to Philo Farnworth, the inventor of electronic television and Hirsch ${ }^{[5]}$. (The physics basis for the IEC is essentially the same as for the CRIEC).

Corresponding Author: George H. Miley, Department of Nuclear, Plasma and Radiological Engineering, University of Illinois, Urbana, IL. USA 
The concept was abandoned for many years, until the early 1990's, when a modified version of the IEC device was developed at the University of Illinois. In this modified device, the ion guns were replaced with a grid-produced plasma discharge, operating in the unique "star" mode ${ }^{[6]}$. In addition to the spherical geometry used in the earlier devices, cylindrical versions have been developed at the University of Illinois that form the basis for the application presented in this work.

In the spherical design, a transparent grid is biases at 60 to $90 \mathrm{kV}$. The grid acts as a cathode relative to the grounded vacuum vessel wall. When the vessel is filled with deuterium gas, a discharge occurs between the wall and the high-voltage cathode grid. The ions produced in the discharge are extracted from the plasma by the cathode grid, accelerated and focused at the center of the sphere where nuclear fusion reactions primarily occur. The grid provides re-circulation of the ions, increasing the power efficiency. In the high current regime, an electric potential structure develops in the non-neutral plasma, creating virtual electrodes that further enhance ion containment and recirculation $^{[6]}$ (Fig. 1). Experimental measurements have demonstrated the existence of such potential structures, but at considerably lower currents than those required for higher yields. Potential structure stability could be an issue when high currents are used, although theoretical studies have not identified a problem to date.

The spherical IEC units currently in operation produce $\sim 10^{7} 2.54-\mathrm{MeV}$ neutrons/s at steady state, due to the fusion of two deuterium atoms (DD). Pulsed operation has achieved up to $10^{9}$ neutrons/s. This DD yield is equivalent to $10^{11} \mathrm{n} / \mathrm{s}$ if a mixture of deuterium/tritium (DT) is used under similar conditions, the scaling being proportional to the respective cross section ratio. The present devices have greatly enhanced the understanding of the plasma discharge physics involved and offer an attractive lowlevel neutron source for applications such as a neutron activation analysis (NAA) ${ }^{[7]}$. Indeed, a version of the IEC is being produced commercially as a portable neutron source for industrial NAA applications.

Present steady-state IEC units produce $\sim 10^{8} \mathrm{DD}$ $\mathrm{n} / \mathrm{s}$, while advanced pulsed versions extend to $10^{10} \mathrm{n} / \mathrm{s}$, equivalent to $10^{12} \mathrm{n} / \mathrm{s}$ if DT is used under similar conditions. The present devices have greatly enhanced the understanding of the discharge physics involved and from a practical point-of-view offer an offer an attractive low-level neutron source for applications such as a neutron activation analysis (NAA) ${ }^{[7,8]}$.

The concept of using a spherical IEC directly to drive a sub-critical reactor was proposed earlier and this approach appeared to have merit even before introducing the added advantages of the cylindrical IEC version. The earlier gridded spherical approach employed special laminar grids (vs. wires used in present experiments) to enhance the grid transparency, hence unit efficiency ${ }^{[9,10]}$ by a factor 10 (theoretically up to a factor 100). A further improvement is by using sealed units and to operate the high-voltage grids inductively by AC supply through a transformer supply ${ }^{[11]}$.
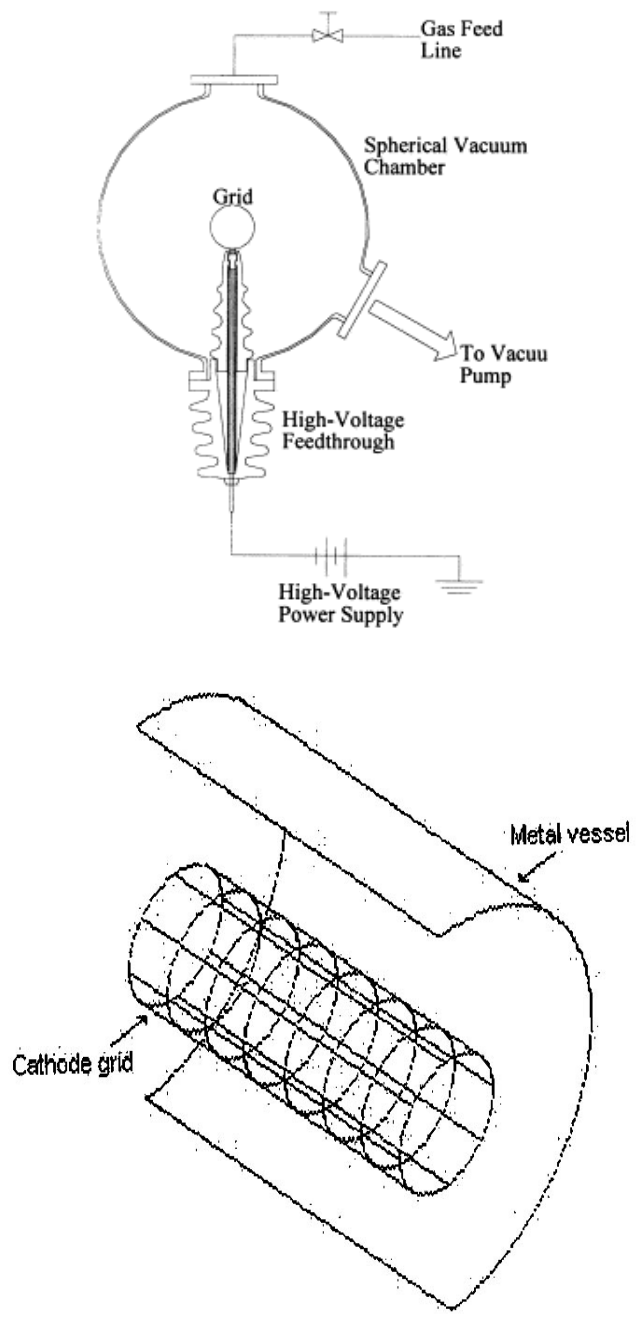

Fig. 1: Spherical IEC design (up) and cylindrical design (below)
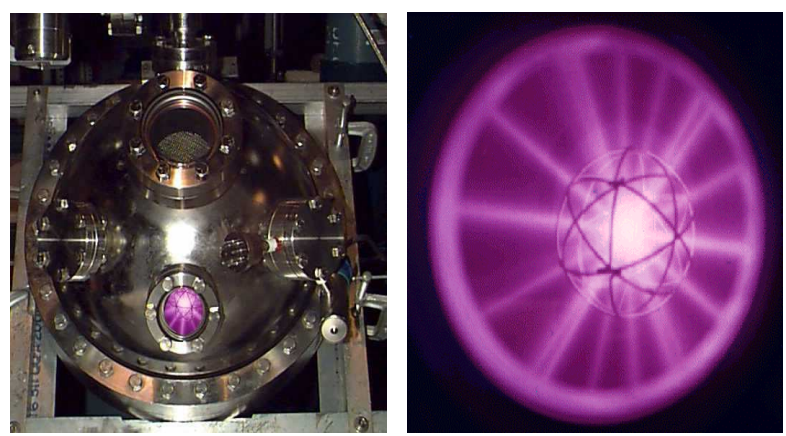

Fig. 2: Arrangement of the "N-Device" IEC. The potential field associated with this type of grid causes the "star mode" ion beams that are clearly visible in the photo 


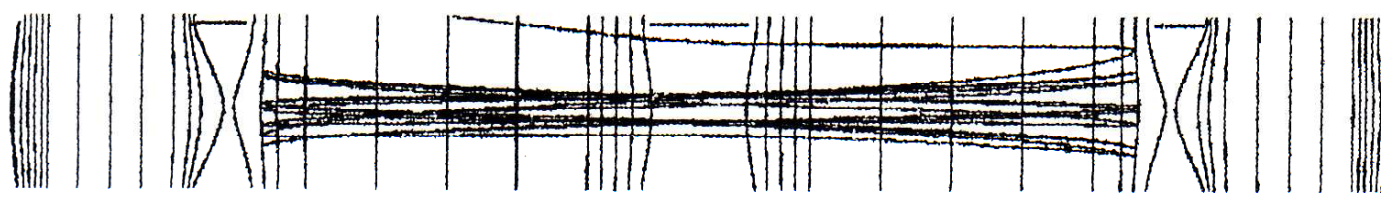

Fig. 3: Diagram of the C-device with calculated ion trajectories and equi-potential surfaces

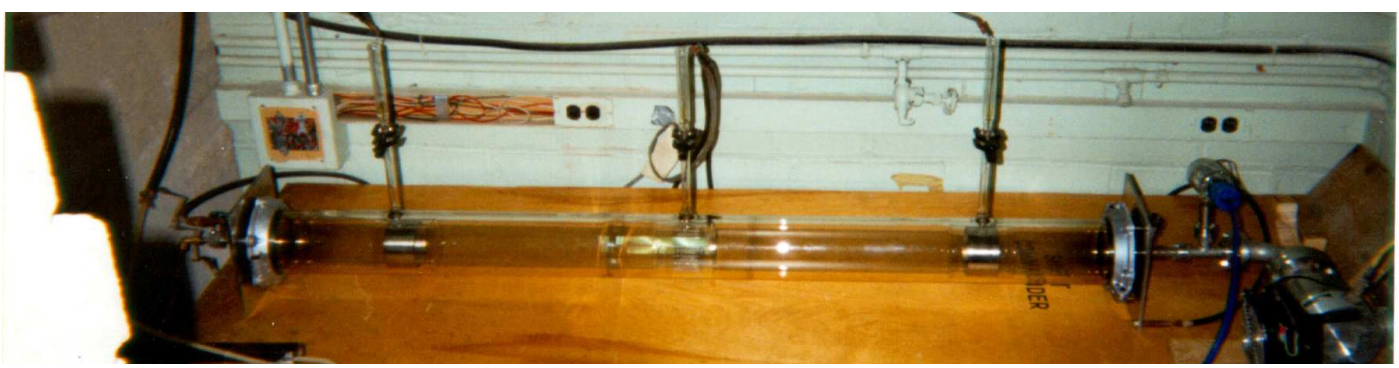

Fig. 4: Photograph of an earlier cylindrical IEC experiment capable of $10^{7} \mathrm{D}-\mathrm{D} \mathrm{n} / \mathrm{s}$

However, grid transparency issues are avoided by the use of hollow electrodes in the unique cylindrical RCIEC design. That, along with the ability of the RC-IEC to produce a long line-type neutron source (vs. the point-type IEC is particularly attractive. An example of the spherical IEC out star mode operation is shown in Fig. 2.

\section{CYCLINDRICAL IEC'S}

While most IEC research to date has involved spherical devices, cylindrical IECs ${ }^{[12]}$ offer many advantages in a variety of practical applications, including the present sub-critical reactor system. The cylindrical geometry is advantageous in a wide range of engineering configurations that require coverage of a broad area with neutrons. It is also capable of more efficient heat rejection than the gridded spherical unit, since rejected heat is carried by the larger area hollow electrodes vs. thin-line or plates.

The prototype cylindrical IEC version (Fig. 3 and 4), called a C-device, represents a prototype of the RCIEC envisioned for the driven sub-critical application. It forms deuterium (or deuterium-tritium) beams in a hollow cathode configuration such that fusion occurs along the extended colliding beam volume in the center of the device, giving a line-type neutron source. The prototype design uses hollow cylindrical anodes (held at ground potential) at either end of the unit, while a similar, but longer hollow cylindrical cathode in the center of the device is biased to a high negative potential. Deuterium gas introduced at the end of the unit is ionised in the resulting discharge, creating an ion source. These ions are accelerated back and forth along the axis of the unit, where they collide and fuse. The prototype C-device shown in Fig. 4, uses three electrodes placed in a cylindrical glass vacuum chamber having a diameter of $\sim 8 \mathrm{~cm}$ and length $\sim 100 \mathrm{~cm}$. The center cathode is constructed from a hollow, thin-wall, stainless steel tube. The two anodes are also hollow steel tubes, held at a large positive (about $+80 \mathrm{kV}$ ). Positive ions formed in the plasma between the electrodes are accelerated toward the center cathode. Because the anodes and cathodes are hollow, most ions and electrons pass through them without colliding with the structure, giving an effective transparency of $\sim 100 \%$. As ions pass through the center cathode, they are accelerated and focused by the electric fields of the electrodes, forming a narrow beam of energetic ions. A counter-streaming beam is formed by ions reflected at the anode. That is, they effectively stop as they approach the anode, turn around and are reaccelerated back towards the center cathode. The ion density peaks in the beam path, significantly enhancing the fusion rate along the interacting beams.

Figure 3 also shows a sample ion trajectory plot calculated for the prototype C-device. The tightly focussed beams passing through the center cathode are evident from the plot. The tightly focussed beams passing through the centre cathode are evident from the plot. The electrodes at the ends of the chamber, called "reflector" dishes, are solid, concave steel surfaces held at ground potential. The dishes "reflect" and focus electrons toward the center of the anodes where they pass through and recirculate in a manner similar to the ions. Proper design of this feature (and of the electrodes) is essential to prevent space charge expansion of the ion beams. The design of the $\mathrm{C}$-device was based on extensive trajectory plots such as these shown, where the electrode configuration and design was varied. A more refined version employed additional electrodes for improved beam control; however, the basic principles remain the same as illustrated here.

In the present experimental device, the resulting line neutron source covers approximately $40 \mathrm{~cm}$ of 
elongated fusion zone. The device dimensions and construction were selected for optimal flexibility when changing electrode designs (Fig. 4) in the prototype experimental campaigns.

\section{CONCLUSION}

We have demonstrated in long years experiments and theoretical analysis how the IEC neutron generators are working and how the parameters can be reduced for developing a low-cost IEC neutron source (spherical or cylindrical) based on advanced lamellar grids, sealed units and where the power supply for the high grid voltage could be given inductively by transformators operated from the standard power grid or from batteries.

\section{REFERENCES}

1. Miley, G.H., H. Hora, Y. Yang, L. Wu, H. Momota and X. Zhong Li, 2005. Subcritical nuclear reactor using the cylindrical inertial electrostatic confinement neutron source. 13th Intl. Conf. Nuclear Engin. Beijing, May 2005, Paper ICONE 13-50606.

2. Hora, H., M.A. Prelas and G.H. Miley, 2001. Kernspaltreaktor mit unterkritischer Auslegung. German Patent 4327920 C2 (priority 23 Aug. 1993, granted 13 Mar. 2003); H. Hora and G.H. Miley, Upgraded reactor for electrostatic confined nuclei. German Patent Appl. 10118 251.1.

3. Dautray, R., 1993. Position Paper of the CEA, CHC/93-104, 3 Dec.
4. Rubbia, C.C., 1998. Harmless energy from nuclei: A quest worth pursuing? Contribution to the Workshop on innovative Options in the field of Nuclear Fission Energy, Centre de Physique des Houches.

5. Hirsch, R., 1967. Inertial electrostatic confinement of ionised fusion gases. J. Appl. Phys., 38: 45224533.

6. Miley, G.H. et al., 1997. Discharge characteristics of the spherical interial electrostatic confinement (IEC) device. IEEE Trans. Plasma Sci., 25: 733739.

7. Kulcinski, G.L. et al., 1997. Overview of neutron/proton source applications from IEC fusion devices. Trans. ANS, 77: 507.

8. Gu, Y. and G.H. Miley, 2000. Experimental study of potential structure in a spherical IEC fusion device. IEEE Trans. Plasma Sci., 28: 331-346.

9. Hora, H., 1998. The Regensburg team FH-FT. In Innovation, Technology and Economics. S. Roderer, Publisher, Regensburg, Germany, pp: 37.

10. Miley, G.H. et al., 1998. Scaling of the inertial electrostatic confinement (IEC) for near-term thrusters and future fusion propulsion. Proc. 15th Symp. on Space Nuclear Power and Propulsion (STAIF98), Part 3, pp: 1373-1375, Albuquerque, NM.

11. Hora, H., 1996. Germany Patent Appl., 19641471.7.

12. Dolan, T., 1970. Electrostatic inertial plasma confinement. Ph.D Thesis. Department of Nuclear Engineering, University of Illinois at UrbanaChampaign. 